\title{
A New Predictive Flow Control Scheme for Efficient Network Utilization and QoS
}

\author{
Dongyu Qiu and Ness B. Shroff* \\ School of Electrical and Computer Engineering \\ Purdue University \\ West Lafayette, IN 47907-1285, U.S.A. \\ E-mail:\{dongyu, shroff\}@ecn.purdue.edu
}

\begin{abstract}
In this paper we develop a new predictive flow control scheme and analyze its performance. This scheme controls the nonreal-time traffic based on predicting the real-time traffic. The goal of the work is to operate the network in a low congestion, high throughput regime. We provide a rigorous analysis of the performance of our flow control method and show that the algorithm has attractive and useful properties. From our analysis we obtain an explicit condition that gives us design guidelines on how to choose a predictor. We learn that it is especially important to take the queueing effect into account in developing the predictor. We also provide numerical results comparing different predictors that use varying degrees of information from the network.
\end{abstract}

\section{INTRODUCTION}

In an efficiently utilized network, even if the dimensioning of network resources has been done correctly and the admission control mechanism is good, the network may go into periods of congestion due to the transient oscillations in the network traffic. It is thus necessary to develop a mechanism to reduce quickly the congestion or pre-empt it, so as to cause the least possible degradation of quality of service (QoS) to the underlying applications. This is especially important for delay sensitive traffic such as video or voice traffic, which we will refer to as real-time (RT) traffic. For these types of traffic, if a packet arrives after a certain deadline it is assumed to be lost. There are other elastic types of traffic, such as data traffic (e-mail, file transfers, web browsing, etc.), that are more delay insensitive and we will call them non-real-time (NRT) traffic. Our goal will be to develop a predictive flow

\footnotetext{
*Please address correspondence to Ness B. Shroff (shroff@purdue.edu, Tel. +1-765-494-3471, Fax. +1765-494-3358). This work has been partially supported by the National Science Foundation through the NSF CAREER award NCR-9624525, and the Indiana 21st Century Research and Technology Award 1220000634.
}

control algorithm that facilitates high network throughput and low probability of overflow.

A seemingly appealing (but naive) solution to prevent the NRT traffic from affecting the RT traffic is to simply give the RT traffic priority over the NRT traffic. While this ensures that the RT traffic is not delayed by the NRT traffic in the network, it also means that the NRT may have to be retransmitted many times (due to buffer overflows within the network), to be successfully transmitted, thus, reducing the overall throughput in the network. Hence, the problem of controlling the NRT traffic must be more carefully considered.

There have been different research efforts on how to control the NRT traffic arrival rate, from simple single-bit feedback control [5][9] to explicit-rate control [1][2][3][10][17]. Some rate-based control schemes are fairly simple and do not consider the impact of high-priority RT traffic, nor do they consider the round trip propagation delay. In these schemes, low-frequency high-magnitude oscillations may exist in the queueing of packets that occurs within the network. By considering the high-priority traffic and round-trip delays, an explicit-rate control scheme based on the result of frequency domain analysis of multimedia traffic [12] was proposed in [17]. The basic idea of [17] is to apply a lowpass filter on the high-priority traffic, then predict the low frequency part of the high-priority traffic, and finally control the NRT traffic rate. Because of the low-pass filter, the high-frequency oscillations in the queueing process are eliminated, thus improving the stability. In [17], the control objective was to minimize the unused link capacity by minimizing the mean squared prediction error of the highpriority traffic rate. But, the authors in [17], use a simple step function, rather than a stochastic process, to model the smoothed RT traffic, In some cases, this approach could lead to poor performance, as will be illustrated in Section 6 of this paper. Further, modeling the RT traffic with a step function does not shed any light on the queueing behavior (congestion characteristics) at the network node being controlled.

For an explicit-rate control scheme to be practical when high-priority RT traffic (or other uncontrollable traffic) is present, there are two main problems that need to be addressed. 
1. Individual Link-Level Problem: Predicting the link capacity available for the NRT traffic at an individual link in the network. One approach to controlling the NRT traffic is to be able to predict the RT traffic (at the link of interest) at some time in the future. Then, based on this prediction, control the NRT traffic. Prediction is required due to the relatively large propagation delay component (large delay bandwidth product) in high-speed networks. Because of the stochastic variations in the aggregate high-priority RT traffic at each link, the available link capacity for the NRT traffic will be time-varying. Our objective is to find a way to predict the available link capacity for the NRT traffic such that the output link is efficiently utilized and the overflow probability is kept low.

2. Network-Level Problem: Distributing the available link capacity for the NRT flows in the network to maximize throughput (or more generally some utility function), based on appropriate fairness requirements. This problem has been studied when all the traffic is controllable $[4][13][11][16]$. We will briefly discuss issues related to this problem in the context of having both NRT (controlled) and RT (uncontrolled) traffic over the network in Section 5. However, this problem is not the focus of the paper.

One of the main difficulties of the first problem is the nonlinearity in the system. The queueing system that determines the congestion on the link is itself a non-linear system. Additionally, the control system to be implemented is also non-linear, because the controlled NRT traffic rate can never be less than zero. Due to this non-linear property, it is difficult to analyze the performance of the system and analyze the stability of the explicit-rate controlled system. Hence, in some works [2][17], a linear system is used as an approximation to analyze the stability of the controlled system. In contrast, in our work, we will explicitly take into account the non-linearity in both the queueing and the control systems. What we present here is a general prediction and control framework. For example, depending on the information that is available from the network, our predictive flow control scheme can vary from one that uses a simple low pass filter with linear control algorithm to a more complex scheme that takes advantage of per-flow information, potentially improving the performance. In Section 6, we will describe three different approaches that take advantage of different levels of information available to us from the network and have differing levels of complexity and performance.

\section{PREDICTIVE FLOW CONTROL MODEL}

As mentioned in the introduction, in this paper we mainly focus on the individual link-level problem. Hence, we focus on a single multiplexing point in the network which consists of a link and an associated buffer that serve both RT and NRT traffic. This multiplexing point in the network could be an output port of a router/switch or a multiplexer. This multiplexing point is modeled by a work-conserving, discrete-time fluid queue with an infinite buffer. Note that we only require that the service discipline be work conserving and not necessarily FIFO. In fact, to prevent the NRT traffic from affecting the RT traffic, it may be better to

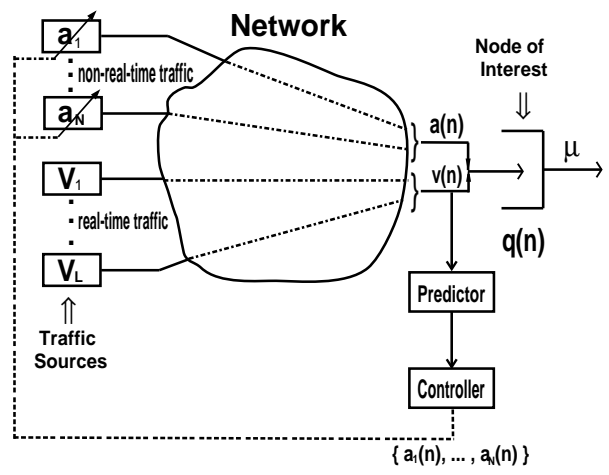

Figure 1: System diagram of predictive flow control

use some kind of priority queue rather than a simple FIFO queue. For analytical tractability, we assume an infinite buffer system. This allows us to obtain valuable insights on how to design a predictive flow control system. We believe that these insights will carry over to finite buffer systems, because of the close relationship of various congestion metrics in finite and infinite buffer systems. For example, it is well known that when the queue level of an infinite buffer system is small, it also implies that the queue level of the corresponding finite buffer system is small. So, if our intention was to develop a control algorithm to maximize the goodput in a finite buffer system with buffer size $x$, one approach would be to develop a control algorithm to minimize the probability that the corresponding infinite buffer queue would exceed a level $x$, given a utilization constraint.

Our system diagram is shown in Fig 1 . Let $V(n)$ be the aggregate amount of RT traffic that arrives at the queue of interest at time $n$. We assume that $V_{\text {max }}:=\sup _{n \geq 0}\{V(n)\}$ is finite and that $V(n)$ is stationary in the mean, i.e., $\bar{V}:=$ $\mathbb{E}\{V(n)\}$.

Our goal is to control the NRT traffic based on predicting the aggregate RT traffic at the queue. We define $a_{i}(n)$ to be the available link capacity for $i$ th NRT traffic computed at time $n$ based on the predicted value of the RT traffic rate. This explicit rate information is sent back to the $i$ th NRT traffic source. Since, in this paper, our main focus is on the individual link-level problem, we assume that the calculated available link capacity for the NRT traffic can be fully utilized, i.e., the NRT traffic sources always have enough data to transmit. Let $N$ be the number of NRT traffic sources and let $n_{i}, i=1, \ldots, N$ be the round trip delay between the $i$ th NRT source and the destination. (Note that we need the notation $n_{i}$ for our theoretical analysis. But in practice, the actual value of $n_{i}$ may or may not be needed. See Section 5 and 6). Then $a(n)=\sum_{i=1}^{N} a_{i}\left(n-n_{i}\right)$ is the aggregate NRT traffic arrival to the queue at time $n$. (We assume that a control message is propagated from the queue of interest to the destination and back to the source). We further define $\hat{V}_{i}(n)$ as the predicted value of $V(n)$ based on the history of $V$ before time $n-n_{i}$. We assume that the predictor is linear. ${ }^{1}$

\footnotetext{
${ }^{1}$ Using a linear predictor has been found to give very good results for video traffic $[12,17]$, and we find that it gives
} 
Let $V(z)$ be the Z-transform of $V(n)-\bar{V}$ and $\hat{V}_{i}(z)$ be the Z-transform of $\hat{V}_{i}(n)-\bar{V}$. Then

$$
\hat{V}_{i}(z)=z^{-n_{i}} H_{i}(z) V(z)
$$

where $H_{i}(z)$ is a causal, stable linear time invariant system [15]. This form of predictor is quite general and includes all time-series types of predictors (e.g. ARMA). From Eq. (1), we can easily show that $\mathbb{E}\left\{\hat{V}_{i}(n)\right\}=\bar{V}$, i.e., the predictor is unbiased.

The workload or queue-length, $q(n)$, at time $n$ at the queue of interest will be determined by $a(n), V(n)$, and the service rate (link rate) of the queue $\mu$. We assume that the queue process begins at time $n=0$ and $q(0)=0$. For stability, we also require that $\bar{V}<\mu$.

The feedback control scheme is as follows. We predict the aggregate $\mathrm{RT}$ traffic rate, and use the predicted value to compute $a_{i}(n), 1 \leq n \leq N$. Here, we ignore the time needed for prediction and computing (however, we can just as easily add it to the delay $n_{i}$ ). In this paper, we will present a predictive flow control algorithm and analyze the queueing behavior of the system. For simplicity of exposition, we will first consider only one NRT traffic source and develop a result for the queueing behavior at the queue of interest. We extend this result to the multiple NRT traffic sources case in Section 4.

\section{SINGLE NRT TRAFFIC MODEL}

\subsection{Predictive Flow Control Algorithm}

In this section, we assume that there is only one NRT traffic source $a_{1}(n)$ (or a group of NRT traffic loops with the same round trip delay $\left.n_{1}\right)$. Note that by definition, $a(n)=a_{1}(n-$ $\left.n_{1}\right)$. Ideally, what we want to achieve is $a(n)+V(n)=\mu$ at all time $n$. However, there are two difficulties in achieving this. Firstly, since we do not know $V(n)$ in advance, we need to estimate its value through prediction which will always have a certain possibility of error. Secondly, $V(n)$ could be greater than $\mu$ but since $a(n)$ cannot be negative, the sum $a(n)+V(n)$ cannot be made equal to $\mu$.

Taking into consideration the possibility of prediction error and the possibility that $V(n)>\mu$, it seems plausible to control the NRT traffic $a_{1}(n)$ such that

$$
a_{1}(n)=\left[p \mu-\hat{V}_{1}\left(n+n_{1}\right)\right]^{+},
$$

where $p$ is the percentage of output link capacity that we would like to utilize $\left(p>\frac{\bar{V}}{\mu}\right)$ and $[x]^{+}=x$, if $x \geq 0$, and $[x]^{+}=0$, otherwise. (Note that in [17], the non-linearity was not considered and a simplified version of Eq. 2, $a_{1}(n)=$ $p \mu-\hat{V}_{1}\left(n+n_{1}\right)$, was used). Although this method of control is easy to implement, it has a major drawback. The drawback is that this type of control does not take into account the queueing behavior, but simply the difference between the service rate and the RT traffic. To elaborate, consider even the situation of perfect prediction, and that $V(n)$ has exceeded $\mu$ for some time. During the period that $V(n)$ has exceeded $\mu$, the above equation correctly sets $a_{1}(n)$ to

good results for other types of traffic as well. Unlike other works in the literature, we allow both the control algorithm and the queueing operation to be non-linear. be zero. But, even after $V(n)$ is no longer larger than $\mu$, there could still be a substantial backlog in the queue, during which $a_{1}(n)$ should be set to zero. However, according to Eq. (2), the moment $V(n)$ is less than $\mu$, the NRT source is allowed to transmit, thus potentially causing unnecessary congestion at the queue. This will also be shown in our simulation results. Hence, we need a better flow control algorithm. What we will attempt to do is to keep the queue length at the node of interest small, while maintaining a certain level of throughput given by

$$
\lim _{n \rightarrow \infty} \frac{\sum_{j=1}^{n}(a(j)+V(j))}{n}=p \mu
$$

Note again, that at time 0 the queue will be empty.

Proposed Control Algorithm ( $N=1$ case)

1. Define a virtual queueing process $q_{1}(n)$ and set $q_{1}(0)=$ 0 .

2. $q_{1}(n)=\left[q_{1}(n-1)+\hat{V}_{1}(n)-p \mu\right]^{+}$. For $n \leq 0$, we let $V(n)=0$.

3. $a_{1}(n)=\left[p \mu-\hat{V}_{1}\left(n+n_{1}\right)-q_{1}\left(n+n_{1}-1\right)\right]^{+}$. For $n \leq 0$, we let $a_{1}(n)=0$.

In the above algorithm, we maintain a virtual queueing system $q_{1}$ with the predicted RT traffic $\hat{V}_{1}(n)$ as input and $p \mu$ as the service rate. Here we can see the difference between our algorithm and the one given by Eq. (2). In Eq. (2), $a(n)=\left[p \mu-\hat{V}_{1}(n)\right]^{+}$. The NRT traffic rate depends only on the predicted RT traffic rate at time $n$. But in our algorithm, $a(n)=\left[p \mu-\hat{V}_{1}(n)-q_{1}(n-1)\right]^{+}$. When computing the NRT traffic rate, we consider both the predicted RT traffic rate at time $n$ and the predicted queue length at time $n-1$. This results in much lower network congestion, as will be illustrated in the paper. Note that although we have used $n_{1}$ in Step 3 of the algorithm, this does not mean that we need to explicitly know the actual value of $n_{1}$, or even an approximation of it. All we need to know is the value of $\hat{V}_{1}\left(n+n_{1}\right)\left(q_{1}\left(n+n_{1}-1\right)\right.$ can be calculated from $\left.\hat{V}_{1}\right)$. To calculate $\hat{V}_{1}\left(n+n_{1}\right), n_{1}$ may or may not be needed, depending on the type of predictor being used. For example, if we choose a simple predictor $H_{1}(z)=0.5+0.5 z^{-1}$. $\hat{V}_{1}\left(n+n_{1}\right)=0.5 V(n)+0.5 V(n-1)$. Here, we can see that the value of $n_{1}$ is not needed to calculate $\hat{V}_{1}\left(n+n_{1}\right)$. In Section 6, we will provide numerical results comparing different predictors (some that explicitly use values of $n_{1}$ and some that do not).

Also note that when $\hat{V}_{1}(n) \leq p \mu$ for all $n, q_{1}(n)$ will always be zero. In this case our control algorithm will reduce to the linear version of Eq. (2), i.e., $a_{1}(n)=p \mu-\hat{V}_{1}\left(n+n_{1}\right)$. But since the high priority RT traffic does not always satisfy this condition, although the linear version is simple, it is not be appropriate, especially for very bursty RT traffic (we will discuss this further in Section 6).

We now focus exclusively on our control algorithm. Hence we consider two queueing systems with $\hat{V}_{1}(n)$ as the RT traffic input. Note that $q_{1}(n)$ and $q_{2}(n)$ described below are not 
the queue lengths of an actual queue at a link, but merely useful descriptors for developing our theoretical analysis.

LEMMA 1. Let $q_{1}(n)$ be the workload at time $n$ in a queueing system with input $\hat{V}_{1}(n)$ and service rate $p \mu$ and let $q_{2}(n)$ be the workload at time $n$ in a queueing system with input $a(n)+\hat{V}_{1}(n)$ and service rate $p \mu$. Then $q_{2}(n)=q_{1}(n)$ for any $n \geq 0$.

\section{Proof: See Appendix A}

From Lemma 1, we observe that the queue length of a system that uses our control mechanism having both $a(n)$ and $\hat{V}_{1}(n)$ as input is the same as the queue length of a system which only has $\hat{V}_{1}(n)$ as input. This is a very desirable property. It means that we can send more data and at the same time not increase the queue length. Of course, in real network, the RT traffic rate will be $V(n)$ rather than $\hat{V}_{1}(n)$, hence we will take this into account as well. Next, we will focus on the actual queue that we wish to control. This queue will have input from both the aggregate RT source $V(n)$ and the controlled NRT source $a(n)$. As mentioned before the service rate of this queue is $\mu$. We will show that using our control algorithm, the output link utilization of the queue of interest is fixed at $p$ (this is what we want from (3)).

Proposition 1. For our predictive flow control algorithm defined in steps $1-3$ earlier, under the condition $\bar{V}<p \mu$, we have

$$
\lim _{n \rightarrow \infty} \frac{\sum_{j=1}^{n} a(j)}{n}=p \mu-\bar{V}
$$

Proof: See Appendix B

Since the input to the queue in consideration is $a(n)+V(n)$, and since $\mathbb{E}\{V(n)\}=\bar{V}$, what Proposition 1 tells us is that the average aggregate input rate to the queue is given by $p \mu$. As mentioned earlier, this is exactly the desired utilization we want. This also tells us that when we fix $p$ in our control algorithm, the output link utilization is also fixed and does not depend on other predictor parameters.

Next, we will fix $p$, and observe how the queue length of the queue of interest will be affected by different predictor parameters.

\subsection{Properties of the Flow Controlled Queue}

The queue in consideration has service rate $\mu$ (output link rate) and the total input to it is $a(n)+V(n)$ at time $n>$ 0 , where $V(n)$ is the aggregate RT input and $a(n)$ is the resultant NRT input as determined through our predictive flow control algorithm. As mentioned before, $q(n)$ denotes the workload or queue length of this queue.

Since the queue is empty at time $n=0, q(n)$, the queue length at time $n$, can be expressed as [8] [14]:

$$
q(n)=\sup _{0 \leq n_{0} \leq n}\left\{\sum_{j=n_{0}+1}^{n}(a(j)+V(j)-\mu)\right\}
$$

In this section, we will analyze the relationship between $q(n)$ and the queue length $q_{0}(n)$ of a queueing system with only RT traffic input $V(n)$. The next theorem shows that our predictive flow control algorithm can achieve a queue length $q(n)$ that is only an additive constant $\left(2 C_{1}\right)$ larger than $q_{0}(n)$, for any $n$. Here, $q_{0}(n)$ is defined as the queue length at time $n$ of a queueing system $q_{0}$ with arrival rate $V(n)$ and service rate $\mu$. Note that $q_{0}$ has the same RT arrival rate $V(n)$ as in our controlled system, but has no NRT input!

THEOREM 1. Under the definitions and predictive flow control algorithm defined above, if $V_{\max }<\infty$ and $\frac{p \mu-\bar{V}}{\mu-\bar{V}} \leq$ $H_{1}(1) \leq 1$, we have $q(n) \leq q_{0}(n)+2 C_{1}$, where $C_{1}$ is a constant that does not depend on $n$.

\section{Proof: See Appendix C}

What Proposition 1 tells us is that we can completely achieve the the utilization constraint that we set to achieve in Eq. (3) Now what Theorem 1 tells us is that under the condition $\frac{p \mu-\bar{V}}{\mu-\bar{V}} \leq H_{1}(1) \leq 1$, the queue length at any time in our controlled queue will at most exceed the queue length in a queueing system $q_{0}$ by a constant. This is quite remarkable since $q_{0}$ is a queueing system that has no NRT traffic input at all and hence one would expect that its queue length would be a very loose lower bound on the queue length generated by a control scheme that attempts to achieve a utilization of $p \mu>\bar{V}$. However, even when the $q(n)$ of our scheme is compared to $q_{0}(n)$ the queue length without NRT traffic, we can show that $q(n)$ is only an additive constant (hence not dependent on the time $n$ ) larger than $q_{0}(n)$.

We are now ready to extend this result to the case when multiple NRT traffic sources with different round trip delays are multiplexed at the node of interest.

\section{MULTIPLE NRT TRAFFIC MODEL}

We now consider a system with $N$ NRT traffic sources. Remember that $\hat{V}_{i}(n)$ is the predicted value of the aggregate RT traffic $V(n)$ based on the history of $V$ before time $n-n_{i}$. Again, note that $q(0)=0$, our predictive flow control algorithm is given as follows:

\section{Proposed Control Algorithm}

1. Set $q_{i}(0)=0,1 \leq i \leq N$.

2. $q_{i}(n)=\left[q_{i}(n-1)+\hat{V}_{i}(n)-p \mu\right]^{+}$. For $n \leq 0$, we let $V(n)=0$.

3. $a_{i}(n)=\frac{1}{N}\left[p \mu-\hat{V}_{i}\left(n+n_{i}\right)-q_{i}\left(n+n_{i}-1\right)\right]^{+}$. For $n \leq 0$, we let $a_{i}(n)=0$.

From the proof of Proposition 1 and the above algorithm, it follows that $\lim _{n \rightarrow \infty} \frac{\sum_{j=1}^{n} a_{i}(j)}{n}=\frac{1}{N}(p \mu-\bar{V})$ for the multiple NRT traffic case. Note that how to assign the available link capacity is in fact a network-level problem. Since we only consider a single link here, for fairness, in Step 3, we assign the available link capacity in such a way that each NRT traffic has the same average arrival rate. This is by no means 
a necessary condition, and can be relaxed by modifying step 3 above in favor of a more unfair system if the situation or the solution of the network-level problem requires it. We will discuss this further in Section 5 .

We now have a version of Theorem 1 with multiple NRT sources having different round-trip delays. Once again let $q_{0}(n)$ correspond to the queue length at time $n$ of a queueing system with service rate $\mu$ and input $V(n)$, while $q(n)$ is the queue length at time $n$ of the system with input $V(n)+a(n)$, where $a(n)=\sum_{i=1}^{N} a_{i}\left(n-n_{i}\right)$.

THEOREM 2. With the definitions and predictive flow control algorithm defined above, If $V_{\max }<\infty$ and $\frac{p \mu-\bar{V}}{\mu-\bar{V}} \leq$ $H_{i}(1) \leq 1$ for all $1 \leq i \leq N$, there exists a constant $C$ such that $q(\bar{n}) \leq q_{0}(n)+\overline{2} C$ for any $n \geq 0$.

\section{Proof: See Appendix D}

As before, $q_{0}(n)$ is the queue length without the NRT traffic. Therefore, Theorem 2 tells us that with our predictive flow control algorithm, the queue length of the system will be less than the queue length with only RT traffic plus a constant finite value that does not depend on $n$.

\section{DISCUSSION}

Consider the condition $\frac{p \mu-\bar{V}}{\mu-V} \leq H_{i}(1) \leq 1$ that is obtained from our main results. We can see that when $H_{i}(1)=1$, this condition is satisfied for any $p \leq 1$. Hence, from now on the first requirement for our predictor will be that $H_{i}(1)=1$. Next, we provide more justification why designing a predictor under this condition is desirable.

\subsection{Tail of Queue Length Distribution (Tail Probability)}

From Theorem 2, we know that the only requirement for the $\mathrm{RT}$ traffic is $V_{\max }<\infty$. So, the result can be applied to both short range dependent and long range dependent $\mathrm{RT}$ traffic.

Now, consider the probability $\mathbb{P}\{q(n)>x\}$, when $x$ is large. Since $q(n) \leq q_{0}(n)+2 C$, we have,

$$
\mathbb{P}\{q(n)>x\} \leq \mathbb{P}\left\{q_{0}(n)>x-2 C\right\} .
$$

Let $\mathbb{P}\left\{Q_{0}>x\right\}=\lim _{n \rightarrow \infty} \mathbb{P}\left\{q_{0}(n)>x\right\}$ be the steady state tail of the queue length distribution (also usually called the tail probability) of $q_{0}$ and let $\mathbb{P}\{Q>x\}=\lim _{n \rightarrow \infty} \mathbb{P}\{q(n)>$ $x\}$ be the tail probability of $q$. When the aggregate RT traffic $V(n)$ is short range dependent, for a large class of short range dependent traffic [8], we have $\mathbb{P}\left\{Q_{0}>x\right\} \sim$ $A e^{-B x}$ when $x$ is large, where $A, B$ are constants. So, we have,

$$
\lim _{x \rightarrow \infty} \frac{\mathbb{P}\{Q>x\}}{\mathbb{P}\left\{Q_{0}>x\right\}} \leq \lim _{x \rightarrow \infty} \frac{A e^{-B(x-2 C)}}{A e^{-B x}}=e^{2 B C} .
$$

When the aggregate RT traffic $V(n)$ is long range dependent, for a large class of traffic models [6], the tail probability $\mathbb{P}\left\{Q_{0}>x\right\} \approx A e^{-B x^{d}}$, for $x$ large and where $A, B, d$ are constants. Further, $0<d<1$, hence,

$$
\lim _{x \rightarrow \infty} \frac{\mathbb{P}\{Q>x\}}{\mathbb{P}\left\{Q_{0}>x\right\}} \leq \lim _{x \rightarrow \infty} \frac{A e^{-B(x-2 C)^{d}}}{A e^{-B x^{d}}}=1
$$

Since the right hand side of Eqs. (6) and (7) is a constant (1 for Eq. (7)), this tells us that the tail probability for the flow controlled queue does not diverge from the queue length distribution of the system with only RT traffic. Since the tail probability in infinite buffer systems is intimately connected to the overflow probability in finite buffer systems, this again makes a rigorous statement on the effectiveness of our flow control scheme.

We have show that when $\frac{p \mu-\bar{V}}{\mu-V} \leq H_{i}(1) \leq 1$ for all $1 \leq$ $i \leq N$, the queue length of the predictive flow control system will not exceed the original queue length with only RT traffic plus a constant value. Now, the question is what happens when $H_{i}(1)$ does not satisfy this condition? It turns out that one can easily construct many cases when, if this condition is not satisfied, $\lim _{x \rightarrow \infty} \frac{\mathbb{P}\{Q>x\}}{\mathbb{P}\left\{Q_{0}>x\right\}}$ will go to $\infty$. Due to space limitations, we will not explicitly provide such examples here, however, in our numerical section, this will be illustrated through the help of Figure 5 .

\subsection{Stability}

Another important issue in explicit rate feedback flow control is the stability of the controlled queueing system. From our main result, it follows that if $H_{i}(z)$ are stable and $H_{i}(1)=$ 1 for all $i$, then the queue length will not exceed that of a queue with only RT traffic plus a constant value. This means that if the queue with only RT traffic is stable, our queueing system will also be stable. Further, this stability does not depend on the predictor parameters, once $H_{i}(1)=1$ is known. In a real system, the round trip delays of NRT traffic are not always easily determined and in most feedback flow control schemes, this is an important factor that may cause the system to become unstable. However, in our system, if we keep $H_{i}(1)=1$, the stability will be maintained regardless of errors in estimating the round-trip delay.

\subsection{Per-Flow Information}

As discussed in Section 3, in our predictive flow control scheme we may or may not choose to use the round-trip delay information in computing the parameters of the predictor. This is important because the round trip delay is the only per-flow information in our predictive flow control scheme. Our main result, $H_{i}(1)=1$, tells us that a class of predictors satisfying this condition, will perform well. However, how to choose a predictor from this class is still an open problem. It depends very much on how much information we can infer from the network. For this reason, in the numerical examples (Section 6), we will numerically study predictors that require per-flow information and those that do not.

\subsection{Minimizing which error?}

An important idea that motivates the development of our predictive control method is that minimizing the error at each point in the prediction is less important than minimizing the accumulated error. For example, the minimum mean squared error (MMSE) predictor is a widely used predictor that can be used to predict the RT traffic. In this 
way, we have a minimum mean squared error at each prediction point. However, we know that a large queue length is mainly caused by the accumulated traffic in a time interval. Hence, a minimum error at each point in time may be less important than a minimum accumulated error over the time period in which the queue builds up. This is where the condition $H_{i}(1)=1$ is important. Because it tells us that if, in our predictor, we choose $H_{i}(1)=1$, we are ensuring that the accumulated error is bounded (e.g. Theorem 2). This is also demonstrated by our numerical results which show that the MMSE predictor results in poor performance compared to predictors that maintain $H_{i}(1)=1$. In fact, what we will show is that although the MMSE predictor requires per-flow information, it does not perform as well as predictors that explicitly consider the queueing behavior (i.e., using $H_{i}(1)=1$ ) but do not require per-flow information.

\subsection{Network-Level Problem and Applicability}

As mentioned in the introduction, there are two main problems to be considered for performing explicit-rate flow control. The first problem is at the level of an individual link and the second problem is at the level of the entire network. In this paper, we have focused on the individual link-level problem and predicted the amount of available link capacity for the NRT traffic in the presence of the high-priority RT traffic. The question to be answered now is how to distribute this available capacity in a fair and optimal manner from the point of view of the entire network? This remains an open problem.

However, when the available link capacity is fixed (i.e., there is no uncontrollable or RT traffic in the network), there are very elegant solutions to this problem (e.g. [4], [13], [11], [16]). Our work could be combined with these works to form a complete solution for the explicit-rate flow control problem. This would also make the work applicable to current technologies such as TCP and ATM. In particular, since, our flow control algorithm is a type of explicit rate flow control, it can directly be applied to ATM/ABR type feedback flow control mechanisms. It can also be applied to TCP with the introduction of Active Queue Management (AQM) strategies such as Explicit Congestion Notification (ECN) [7] and Random Exponential Marking (REM). For example, REM is a practical implementation of the network-level optimal flow control algorithm in [13]. Our work could then be combined with REM by simply replacing the fixed available link capacity, by a time-varying link capacity as determined by our predictive flow control algorithm. However, because the available link capacity is time-varying now, many new open problems will arise. For example, the fairness and optimality considered in [13] may not hold now. This will form the basis for our future work in this area.

\section{NUMERICAL RESULTS}

In this section, we assume that $V(n)$ is wide sense stationary. We also assume that $H_{i}(z)$ is of the form $\sum_{m=0}^{M_{i}} B_{m}^{(i)} z^{-m}$. From the discussion above, we know that if we design predictors that do not satisfy the condition $\frac{p \mu-\bar{V}}{\mu-V} \leq H_{i}(1) \leq 1$, the tail probability of the queue length may be much greater than that of predictors which satisfy the condition. Most current works on predictive flow control and even more general feedback flow control focus on minimizing the mean square of some control objective, for example, the mean square of unused link capacity. In [17], a low pass filter is also applied to the high priority traffic to improve the performance and stability. Under this type of method, the predictor may not satisfy the condition $\frac{p \mu-\bar{V}}{\mu-\bar{V}} \leq H_{i}(1) \leq 1$ and in some cases could result in increased congestion. We will demonstrate this through our simulation results. As mentioned in Section 5, since the above condition is satisfied for all $p$ when $H_{i}(1)=1$, the first requirement for our predictor is that $H_{i}(1)=1$. Under the condition that $H_{i}(1)=1$, there are still a number of predictors that we can choose. How to choose an appropriate predictor depends on how much information we can infer from the network. We will discuss three approaches to designing the predictors under the condition of $H_{i}(1)=1$.

Predictor A1: The first predictor we use is very simple. We simply choose a fixed low pass filter $H_{L P F}(z)$ as the predictor. This is in fact the predictor used in the $\mathrm{H}_{2}$ scheme of [17]. Note that although the $H_{2}$ scheme uses the same predictors as ours, it does not use the same control algorithm as ours. In [17], it is assumed that $\hat{V}_{1}(n) \leq p \mu$ at all time $n$ and a linear version of Eq. (2), $a_{1}(n)=p \mu-\hat{V}_{1}\left(n+n_{1}\right)$, is used to calculate the available link capacity. This could result in poor performance, when the non-linear control Eq. (2) is applied, as shown in Figure 6 of this Section.

So, for predictor A1, we let $H_{i}(z)=H_{L P F}(z)$. It satisfies the condition $H_{i}(1)=1$.

Predictor A2: In designing predictor A2, we assume that we can obtain information about both the NRT traffic and the aggregate RT traffic. Hence, here we assume that per-flow information will be available. Again, for simplicity, we first consider the case of one NRT traffic source. From the proof of Theorem 1, we know that the upper bound on $q(n)$ is:

$$
q(n) \leq q_{0}(n)+\sup _{0 \leq n_{0} \leq n} \sum_{j=n_{0}+1}^{n} \Delta(j)-\inf _{0 \leq n_{0} \leq n} \sum_{j=n_{0}+1}^{n} \Delta(j)
$$

Let $l=n-n_{0}$ and define

$$
X_{n, l} \triangleq \sum_{j=n-l+1}^{n} \Delta(j)=\sum_{j=n_{0}+1}^{n} \Delta(j)
$$

Since $\mathbb{E}\left\{X_{n, l}\right\}=0$, we will consider $\operatorname{Var}\left\{X_{n, l}\right\}$.

$$
\begin{aligned}
\operatorname{Var}\left\{X_{n, l}\right\} & =\operatorname{Var}\left\{\sum_{j=n-l+1}^{n} \Delta(j)\right\} \\
& =\sum_{j 1=n-l+1}^{n} \sum_{j 2=n-l+1}^{n} C_{\Delta}(j 1-j 2) \\
& =\sum_{j 1=-l+1}^{0} \sum_{j 2=-l+1}^{0} C_{\Delta}(j 1-j 2)
\end{aligned}
$$

where $C_{\Delta}(j)$ is the auto-covariance function of $\Delta(n)$. Because of the stationarity of $V(n), \operatorname{Var}\left\{X_{n, l}\right\}$ does not depend on $n$. Next, we will minimize $\lim _{l \rightarrow \infty} \operatorname{Var}\left\{X_{n, l}\right\}$ under the condition $H_{1}(1)=1$. The intuition for minimizing $\lim _{l \rightarrow \infty} \operatorname{Var}\left\{X_{n, l}\right\}$ is that this asymptotic variance is related to the congestion in the system (namely $\mathbb{P}\{Q>x\}$ ) [6]. De- 
fine

$$
\delta_{k}=-2 \sum_{m=-(k-1)}^{k-1}(k-|m|) C_{v}(m),
$$

where $C_{v}(m)$ is the auto-covariance function of $V(n)$. We get

$$
\begin{aligned}
\lim _{l \rightarrow \infty} \operatorname{Var}\left\{X_{n, l}\right\}= & -\sum_{m=0}^{M_{1}} B_{m}^{(1)} \delta_{n_{1}+m} \\
& +\sum_{m_{1}=0}^{M_{1}} \sum_{m_{2}=m_{1}+1}^{M_{1}} B_{m_{1}}^{(1)} B_{m_{2}}^{(1)} \delta_{m_{2}-m_{1}}
\end{aligned}
$$

Define the Lagrangian function as

$$
\begin{aligned}
& f\left(B_{0}^{(1)}, \cdots, B_{M_{1}}^{(1)}, \lambda\right)=-\sum_{m=0}^{M_{1}} B_{m}^{(1)} \delta_{n_{1}+m} \\
& \quad+\sum_{m_{1}=0}^{M_{1}} \sum_{m_{2}=m_{1}+1}^{M_{1}} B_{m_{1}}^{(1)} B_{m_{2}}^{(1)} \delta_{m_{2}-m_{1}}+\lambda\left(\sum_{m=0}^{M_{1}} B_{m}^{(1)}-1\right)
\end{aligned}
$$

From $\frac{\partial f}{\partial B_{m}^{(1)}}=0, \quad m=0, \cdots, M_{1}$ and $\frac{\partial f}{\partial \lambda}=0$, we get,

$$
\left[\begin{array}{cccc}
0 & \cdots & \delta_{M_{1}} & 1 \\
\delta_{1} & \cdots & \delta_{M_{1}-1} & 1 \\
\vdots & \ddots & \vdots & \vdots \\
\delta_{M_{1}} & \cdots & 0 & 1 \\
1 & \cdots & 1 & 0
\end{array}\right]\left[\begin{array}{c}
B_{0}^{(1)} \\
B_{1}^{(1)} \\
\vdots \\
B_{M_{1}}^{(1)} \\
\lambda
\end{array}\right]=\left[\begin{array}{c}
\delta_{n_{1}} \\
\delta_{n_{1}+1} \\
\vdots \\
\delta_{n_{1}+M_{1}} \\
1
\end{array}\right]
$$

From Eq. (8), we can calculate $B_{0}^{(1)}, B_{1}^{(1)}, \cdots, B_{M_{1}}^{(1)}$, and we are done. In the case of multiple NRT sources, we can still use Eq. (8) to calculate $H_{i}(z)$. What we need to do now is to replace $M_{1}$ with $M_{i}, n_{1}$ with $n_{i}$, and $B_{0}^{(1)}, \cdots, B_{M_{1}}^{(1)}$ with $B_{0}^{(i)}, \cdots, B_{M_{i}}^{(i)}$.

From Eq. (8), it is immediately apparent that the per-flow information (i.e., round trip delay $n_{i}$ ) is required. Hence, for different NRT traffic loops with different round trip delays, we need to have different predictors. This approach is the most complex of the three approaches.

Predictor A3: In predictor A3, we still use Eq. (8) to calculate the predictor parameters. But rather than try to estimate a round trip delay for each NRT flow, we roughly estimate a round trip delay $n_{0}$ for all NRT flows. Once the round trip delay $n_{i}$ is fixed to $n_{0}$, Eq. (8) will be the same for all NRT flows. Thus, as in A1, only one predictor is needed for all NRT traffic flows (this also implies that only one virtual queue needs to be maintained). Since A3 also does explicit prediction based on the stochastic properties of the RT traffic, rather than just use smoothing, we expect that A3 will perform better than A1. In fact, what we will demonstrate through our numerical results is that the performance of A3 (that does not require per-flow information) approximates the performance of A2 (requires per-flow information) quite well.

Under the condition $H_{i}=1$, there are still other approaches to choose the predictors. For example, in the above approaches, we have only considered the stochastic properties of the RT traffic up to two moments. Higher order moments

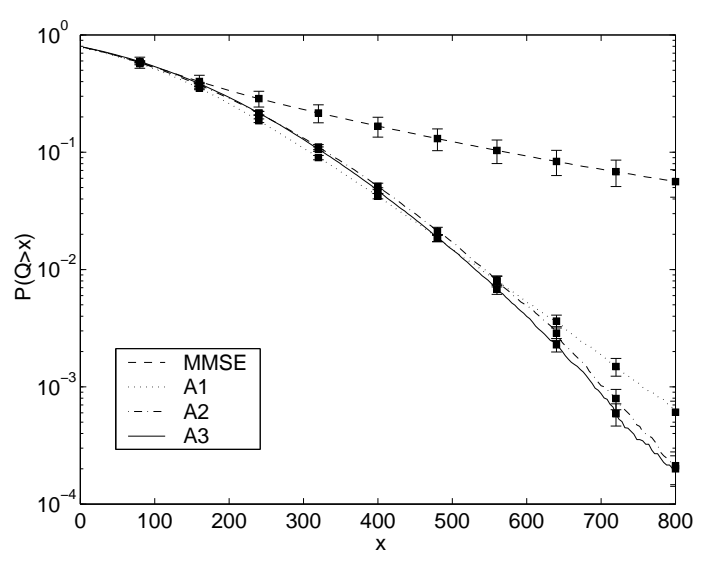

Figure 2: Tail probability with MPEG video traces as RT traffic

and distributions are not considered, primarily because of the complexity this would entail in terms of measurements, in practice. However, given more information on the RT traffic, for example, an accurate model for the RT traffic, one can always design a better predictor.

In the following simulations, we will use the control algorithm defined in section 3.1 (single NRT source case) and in section 4 (multiple NRT sources case). From Proposition 1 , we know that once $p$ is fixed, the output link utilization will also be fixed. In this section, we will compare the performance of the different predictors under the same output link utilization. The predictor designed with minimizing mean square method is represented as MMSE. The MMSE predictor is designed as follows. First, a low pass filter is applied to the high-priority RT traffic. Here, we use a simple move average filter $H_{L P F}(z)=\frac{1}{4}\left(1+z^{-1}+\right.$ $\left.z^{-2}+z^{-3}\right)$ as the low pass filter. Next, a standard minimizing mean square error linear predictor $H_{M M S E}(z)$ with the form $\sum_{m=0}^{M_{i}} B_{m}^{(i)} z^{-n_{i}-m}$ is calculated based on the low frequency part of the RT traffic. And the final MMSE predictor is $z^{-n_{i}} H_{i}(z)=H_{L P F}(z) H_{M M S E}(z)$. Note that $H_{M M S E}(z)$ will require explicit knowledge of the round trip delays (i.e., per-flow information).

In $\mathrm{A} 1$, the fixed low pass filter is chosen as $H_{L P F}(z)=$ $\frac{1}{4}\left(1+z^{-1}+z^{-2}+z^{-3}\right)$. In A2, A3, and MMSE predictors, $M_{i}$ is set to 5 . Our simulation results are shown in Figs. 2-4. In these simulations, five NRT sources are simulated and the round trip delays are varied from 5 to 9 . In A3, the roughly estimated round trip delay $n_{0}$ is set to 5 .

Fig. 2 shows the result of a simulation generated with actual MPEG-video traces as the $\mathrm{RT}$ traffic input. The mean rate of the aggregate RT traffic is $11.6 \mathrm{Mbps}$. We set the output link rate $\mu=15 \mathrm{Mbps}$ and the utilization $p=98 \%$. From this figure, we can see that A1, A2, and A3 predictors all have comparable performance, but are all much better than the traditional MMSE predictor.

In Fig. 3, $V(n)$ is a generated Gaussian process which is multi-time scale correlated with $C_{v}(k)=479.599 \times 0.999^{|k|}+$ 


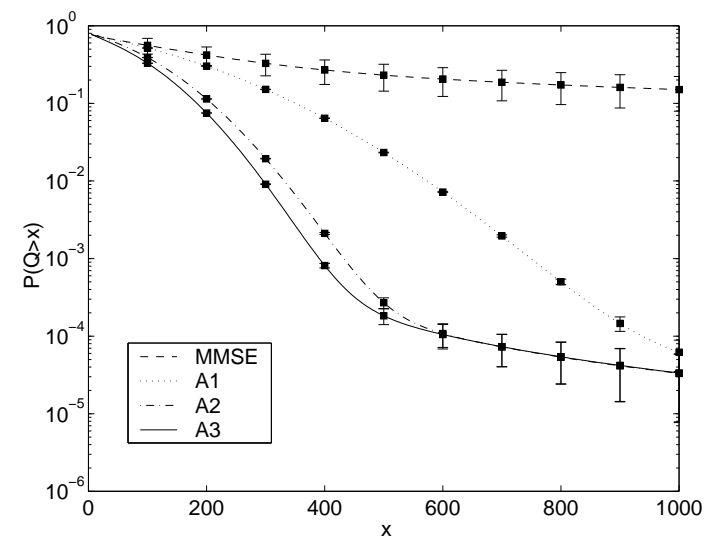

Figure 3: Tail probability with Gaussian process as RT traffic

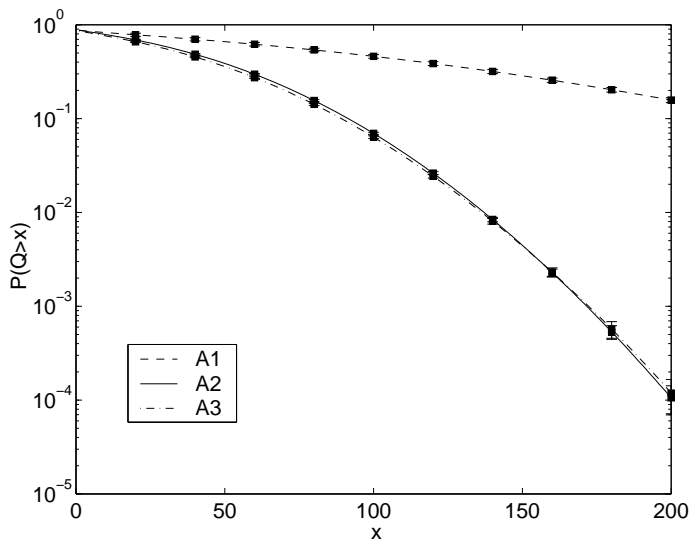

Figure 4: Tail probability with voice sources modeled by exponential on-off processes as RT traffic

$161.787 \times 0.99^{|k|}+498.033 \times 0.9^{|k|}$ and $\bar{V}=100$. The link capacity is 200 , and the utilization is set to $98 \%$. In this case, $V_{\text {max }}$ is no longer finite and our theorem will not hold. However, even in this case, we can observe from Fig. 3, that our predictors significantly outperform the MMSE predictor. In this case, predictors A2 and A3 have similar performance and both outperform A1, especially for small values of the buffer level $x$.

In Fig. 4, 1000 multiplexed voice sources correspond to the aggregate RT traffic. Each voice source is modeled by a Markov modulated On-Off fluid process. The state transition matrix and rate vector are given as follows.

$$
\begin{gathered}
\text { State transition matrix : } \quad\left[\begin{array}{cc}
0.9833 & 0.01677 \\
0.025 & 0.975
\end{array}\right] \\
\text { Input rate vector : }\left[\begin{array}{c}
0 \\
0.85
\end{array}\right]
\end{gathered}
$$

The mean rate of the aggregate $\mathrm{RT}$ traffic is 341.2 . We set the link capacity to 500 and the utilization to $99.8 \%$. In this figure, we compare the performance of three predictors

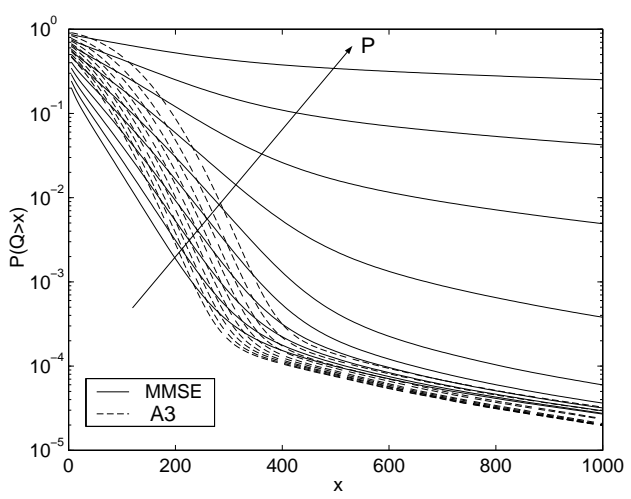

Figure 5: Tail probabilities with different utilization

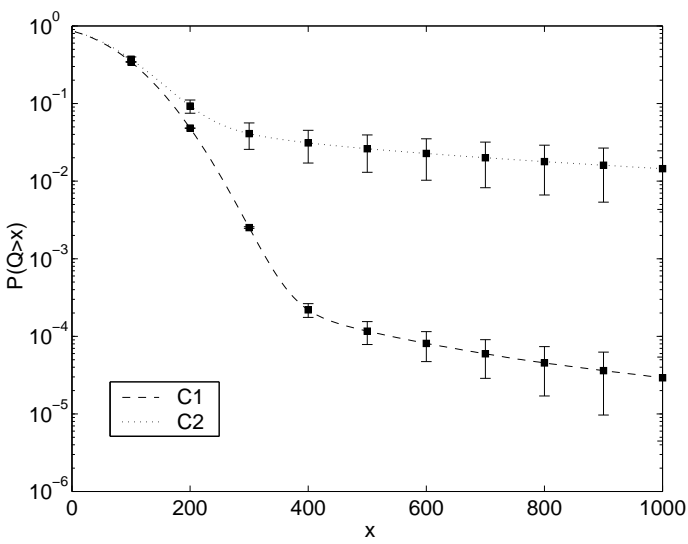

Figure 6: Tail probabilities with different control algorithms

A1, A2, and A3. Since all three predictors satisfy the condition $H_{i}(1)=1$, in most cases, they perform quite well. For example, in the above two figures, A2 and A3 are better than A1, but not by a significant amount. However, in some cases, the difference between the $\mathrm{A} 1$ predictor and the other two predictors can be quite substantial, as is illustrated by Fig 4. This is the case, here, because the A2 and A3 predictors (unlike A1) try to predict the RT traffic at the queue at explicit times in the future, which can be done accurately when the RT traffic is generated from Markovian sources (as is the case, here). We can also see from the above simulations that although predictor A3 does not require per-flow informations, the performance of A3 is almost identical to A2 in most cases. Hence, compared to A1 and A2, the predictor A3 will be a better choice in practical networks.

To explain our main results more clearly (Theorem 1), we do the following simulation. In this simulation, we have only one NRT sources with round trip delay 5. The RT source and the link capacities are the same as in Fig. 3. But we vary the utilization from $90 \%$ to $99 \%$ with a step of $1 \%$. The result is shown in Fig. 5. We can observe that, with an $\mathrm{A} 3$ predictor, the tail probability remains low even when the utilization is very high. But with the MMSE predictor, when the utilization increases, the tail probability also increases. In fact, for the MMSE predictor, $H_{1}(1)=0.878$. 
When $p=94 \%$, we have $\frac{p \mu-\bar{V}}{\mu-V}=0.88$. So, when $p \leq 94 \%$, the tail probabilities of the MMSE and A3 predictor are quite close. However, when $p>94 \%$, the difference is substantial. Hence, our analytical result in Theorem 1 provides a guideline as to when the MMSE predictor will work well and when it would not.

In Fig 6, we compare our control algorithm with the control algorithm that uses Eq. (2). As we mentioned in Section 3, when $\hat{V}_{1}(n) \leq p \mu$ for all $n$, our control algorithm and Eq. (2) reduce to the same linear equation. But when this condition is not true, we can see the difference between these two control algorithms in Fig. 6. In this simulation, there is only one NRT sources with round trip delay 5 .The RT source and the link capacity are still the same as in Fig. 3. To see the difference when the condition $\hat{V}_{1}(n) \leq p \mu$ is violated, we use the same A3 predictor in both control algorithms. In the figure, our control algorithm is marked $\mathrm{C} 1$, and the one that uses Eq. (2) is marked C2. The utilization is set to $98 \%$. But note that when using control algorithm $\mathrm{C} 2$, given $p$, the utilization is not fixed at $p$. In this simulation, we set $p=97.5 \%$ for C2 to make sure that the measured utilization is $98 \%$, the same as C1. From this figure, using the same predictor, we can see that our control algorithm outperforms the one that uses Eq. (2).

\section{CONCLUSION}

In this paper, we first present a predictive flow control model and motivate its development. We then analyze this predictive flow control system allowing both the control and the queueing process to be non-linear. Our analysis gives us insights on what parameters to choose for designing the predictor. Through simulations, we show that our predictive flow control algorithm can significantly outperform the MMSE based predictive flow control algorithm. In fact, even our predictive flow control algorithms that do not require per-flow information also do substantially better than the MMSE based algorithm that uses per-flow information. This tells us that simply minimizing the prediction error without taking into account the queueing in the system, can yield very poor performance.

Through our analysis, we are also able to determine when other predictors in the literature such as the MMSE predictor work well. For example, if a prediction scheme has $H(1)=1$, it is generally expected to perform well. Our main contribution has been in solving the individual-link level problem and to provide a scheme to predict the available link capacity when high priority traffic is present. It can be combined with other solutions for network-level problems to form a complete solution. Our scheme has also been shown to be flexible. For example, depending on the characteristic of the RT traffic, a control algorithm using a virtual queue or a simple linear control algorithm can be chosen. Similarly, depending on how much information we can obtain from the network, there are different approaches that can be used to design the predictor in our framework. In our numerical study, we compare the performance of three such approaches that use differing level of information from the network.

\section{REFERENCES}

[1] E. Altman, F. Baccelli, and J. Bolot. Discrete-Time Analysis of Adaptive Rate Control Mechanisms. High Speed Networks and Their Performance (C-21), pages 121-140, 1994.

[2] E. Altman, T. Basar, and R. Srikant. Congestion Control as a Stochastic Control Problem with Action Delays. Automatica (Special issue on Control Methods for Communication Networks), Dec 1999.

[3] L. Benmohamed and S. Meekov. Feedback Control of Congestion in Store-and-Forward Networks: The case of Single Congestion Node. IEEE/ACM Transactions on Networking, 1(6):693-798, Dec. 1993.

[4] D. Bertsekas and R. Gallager. Data Networks. Prentice Hall, NJ, 1992.

[5] J. Bolot and A. Shankar. Dynamic Behavior of Rate-based Flow Control Mechanisms . ACM Comp. Commm. Review, 20(2):35-49, 1992.

[6] J. Choe and N. B. Shroff. Use of the supremum distribution of Gaussian processes in queueing analysis with long-range dependence and self-similarity. Stochastic Models, 16(2), Feb 2000.

[7] S. Floyd. TCP and Explicit Congestion Notification. ACM Comp. Commm. Review, 24(5), October 1994.

[8] P. W. Glynn and W. Whitt. Logarithmic asymptotics for steady-state tail probabilities in a single-server queue. Journal of Applied Probability, pages 131-155, 1994.

[9] M. Hluchyj and N. Yin. On Closed-loop Rate Control for ATM Networks. In Proc. INFOCOM'94, pages 99-108, 1994.

[10] R. Jain, S. Kalyanaraman, and R. Viswandathan. The OSU Scheme for Congestion Avoidance using Explicit Rate Indication. Technical report, OSU, Sept. 1994.

[11] F. P. Kelly, A. Maulloo, and D. Tan. Rate control for communication networks: Shadow prices, proportional fairness and stability. Journal of Operations Research Society, pages 237-252, March 1998.

[12] S. Li, S. Chong, and C. Hwang. Link Capacity Allocation and Network Control by Filtered Input Rate in High Speed Networks. IEEE/ACM Transactions on Networking, 3(1):10-15, Feb. 1995.

[13] S. H. Low and D. E. Lapsley. Optimization Flow Control, I: Basic Algorithm and Convergence. IEEE/ACM Transactions on Networking, 7(6), Dec. 1999.

[14] R. M. Loynes. The Stability of a Queue with Non-independent Inter-arrival and Service Times. Proc. Cambridge Philos. Soc., 58:497-520, 1962.

[15] A. V. Oppenheim, A. S. Willsky, and S. H. Nawab. Signals \& Systems. Prentice Hall, NJ, 1997.

[16] H. Yaiche, R. R. Mazumdar, and C. Rosenberg. A Game Theoretic Framework for Bandwidth Allocation and Pricing in Broadband Networks. IEEE/ACM Transactions on Networking, 8(5):667-678, Oct. 2000. 
[17] Y. Zhao, S. Li, and S. Sigarto. A linear dynamic model design of stable explicit-rate ABR control scheme. In Proceedings of IEEE INFOCOM, pages 283-292, 1997.

\section{APPENDIX}

\section{A. PROOF OF LEMMA 1}

For $q_{1}(n)$, we have, $q_{1}(0)=0$ and

$$
q_{1}(n)=\left[q_{1}(n-1)+\hat{V}_{1}(n)-p \mu\right]^{+} .
$$

For $q_{2}(n)$, we have, $q_{2}(0)=0$ and

$$
q_{2}(n)=\left[q_{2}(n-1)+\hat{V}_{1}(n)+a(n)-p \mu\right]^{+},
$$

where $a(n)=a_{1}\left(n-n_{1}\right)$. For $n \leq n_{1}, a(n)=a_{1}\left(n-n_{1}\right)=0$, Hence, for $n \leq n_{1}, q_{2}(n)=q_{1}(n)$. For $n \geq n_{1}$, we will use induction to prove $q_{2}(n)=q_{1}(n)$. Assuming that $q_{2}(n-1)=$ $q_{1}(n-1)$, for $n \geq n_{1}$, we have,

$$
a(n)=a_{1}\left(n-n_{1}\right)=\left[p \mu-\hat{V}_{1}(n)-q_{1}(n-1)\right]^{+} .
$$

Next, we will discuss two cases. When $p \mu-\hat{V}_{1}(n)-q_{1}(n-$ 1) $\geq 0$, we have $q_{1}(n)=\left[q_{1}(n-1)+\hat{V}_{1}(n)-p \mu\right]^{+}=0$ and

$$
\begin{aligned}
q_{2}(n)= & {\left[q_{2}(n-1)+\hat{V}_{1}(n)+a(n)-p \mu\right]^{+} } \\
= & {\left[q_{2}(n-1)+\hat{V}_{1}(n)+p \mu-\hat{V}_{1}(n)\right.} \\
& \left.-q_{1}(n-1)-p \mu\right]^{+} \\
= & {\left[q_{2}(n-1)-q_{1}(n-1)\right]^{+}=0 . }
\end{aligned}
$$

When $p \mu-\hat{V}_{1}(n)-q_{1}(n-1)<0, a(n)=0$,

$$
\begin{aligned}
q_{2}(n) & =\left[q_{2}(n-1)+\hat{V}_{1}(n)-p \mu\right]^{+} \\
& =\left[q_{1}(n-1)+\hat{V}_{1}(n)-p \mu\right]^{+} \\
& =q_{1}(n)
\end{aligned}
$$

In both cases, we have

$$
q_{2}(n)=q_{1}(n)
$$

\section{B. PROOF OF PROPOSITION 1}

Throughout this proof, we use the definitions of $q_{1}(n)$ and $q_{2}(n)$ defined in Lemma 1.

For any $j>n_{1}$, we have,

$$
\begin{aligned}
a(j)=a_{1}\left(j-n_{1}\right) & =\left[p \mu-\hat{V}_{1}(j)-q_{1}(j-1)\right]^{+} \\
& =\left[p \mu-\hat{V}_{1}(j)-q_{2}(j-1)\right]^{+} \\
& \geq p \mu-\hat{V}_{1}(j)-q_{2}(j-1) .
\end{aligned}
$$

So,

$$
\begin{gathered}
a(j)+\hat{V}_{1}(j)+q_{2}(j-1)-p \mu \geq 0 \\
q_{2}(j)=\left[q_{2}(j-1)+a(j)+\hat{V}_{1}(j)-p \mu\right]^{+} \\
=q_{2}(j-1)+a(j)+\hat{V}_{1}(j)-p \mu . \\
a(j)=q_{2}(j)-q_{2}(j-1)+p \mu-\hat{V}_{1}(j) \\
=q_{1}(j)-q_{1}(j-1)+p \mu-\hat{V}_{1}(j) .
\end{gathered}
$$

Because $a(j)=0$ for $j \leq n_{1}$, we have,

$$
\begin{aligned}
& \sum_{j=1}^{n} a(j)=\sum_{j=n_{1}+1}^{n} a(j) \\
& \quad=q_{1}(n)-q_{1}\left(n_{1}\right)+\left(n-n_{1}\right) p \mu-\sum_{j=n_{1}+1}^{n} \hat{V}_{1}(j)
\end{aligned}
$$

Next, we focus on $q_{1}(n)-q_{1}\left(n_{1}\right)$. Let $q_{1}$ and $q_{2}$ be the queueing systems with queue lengths $q_{1}(n)$ and $q_{2}(n)$, respectively. Further, let $\mu(n)$ be the amount of traffic that leaves $q_{1}$ at time $n$. We then have,

$$
\begin{aligned}
& q_{1}\left(n_{1}\right)+\sum_{j=n_{1}+1}^{n} \hat{V}_{1}(j)=q_{1}(n)+\sum_{j=n_{1}+1}^{n} \mu(j) \\
& q_{1}(n)-q_{1}\left(n_{1}\right)=\sum_{j=n_{1}+1}^{n} \hat{V}_{1}(j)-\sum_{j=n_{1}+1}^{n} \mu(j) .
\end{aligned}
$$

Because $\bar{V}<p \mu, q_{1}$ will be a stable system. Hence,

$$
\lim _{n \rightarrow \infty} \frac{\sum_{j=n_{1}+1}^{n} \mu(j)}{n}=\lim _{n \rightarrow \infty} \frac{\sum_{j=n_{1}+1}^{n} \hat{V}_{1}(j)}{n}=\bar{V}
$$

i.e.,

$$
\lim _{n \rightarrow \infty} \frac{q_{1}(n)-q_{1}\left(n_{1}\right)}{n}=0 .
$$

So, from Eqs. (10) and (11),

$$
\lim _{n \rightarrow \infty} \frac{\sum_{j=1}^{n} a(j)}{n}=p \mu-\bar{V}
$$

\section{PROOF OF THEOREM 1}

First, we will prove two lemmas that will be needed later to prove Theorem 1.

For the next lemma, we define two queueing systems with service rate $\mu^{\prime}$. The first queueing system has input rate $\hat{V}_{1}(n)$ and corresponding queue length $q_{1}^{\prime}(n)$, while the second queueing system has input rate $a(n)+\hat{V}_{1}(n)$ (where $a(n)$ is determined by our flow control algorithm) and queue length $q_{2}^{\prime}(n)$.

Lemma 2. Under the above definitions, if $\mu^{\prime} \geq p \mu$, we have $q_{2}^{\prime}(n)=q_{1}^{\prime}(n)$, for any $n \geq 0$.

Proof: From Lemma 1 and our algorithm, we know that $a(n)=\left[p \mu-\hat{V}_{1}(n)-q_{1}(n-1)\right]^{+}, n>n_{1}$ and $a(n)=0, n \leq$ $n_{1}$. Now, we define $a^{\prime}(n)$ as, $a^{\prime}(n)=\left[\mu^{\prime}-\hat{V}_{1}(n)-q_{1}^{\prime}(n-1)\right]^{+}$, $n>n_{1}$ and $a^{\prime}(n)=0, n \leq n_{1} . q_{1}$ and $q_{1}^{\prime}$ have the same input traffic rate $\hat{V}_{1}(n)$. But the output link rates are $p \mu$ and $\mu^{\prime}$ respectively. Because $\mu^{\prime} \geq p \mu$, we have $q_{1}(n) \geq q_{1}^{\prime}(n)$ for any $n$. So, we have $a(n) \leq a^{\prime}(n)$ for any $n \geq 0$.

Now, consider a queueing system with $\mu^{\prime}$ as the output link rate and $a^{\prime}(n)+\hat{V}_{1}(n)$ as the input traffic rate. We denote the queue length as $q_{3}^{\prime}(n)$. Comparing $q_{2}^{\prime}$ and $q_{3}^{\prime}$, we find they have the same output link rate. But since $a(n) \leq a^{\prime}(n)$ for any $n$, the input traffic rate of $q_{2}^{\prime}$ will always be less than or equal to the input traffic rate of $q_{3}^{\prime}$. Hence, we have $q_{2}^{\prime}(n) \leq q_{3}^{\prime}(n)$ for any $n$.

Similar to the proof of Lemma 1 , we can also prove that $q_{1}^{\prime}(n)=q_{3}^{\prime}(n)$. Hence, $q_{2}^{\prime}(n) \leq q_{1}^{\prime}(n)$. Also, obviously, $q_{2}^{\prime}(n) \geq q_{1}^{\prime}(n)$. Thus,

$$
q_{2}^{\prime}(n)=q_{1}^{\prime}(n)
$$


It should be noted that the requirement that $\mu^{\prime} \geq p \mu$ is necessary for stability because our objective is to utilize the link at a level $p \mu$.

Lemma 3. Define $D(j)=H_{1}(1)(V(j)-\bar{V})-\left(\hat{V}_{1}(j)-\right.$ $\bar{V})$. If $V_{\max }<\infty$, there exists a constant $C_{1}$ such that $\left|\sum_{j=n_{0}+1}^{n} D(j)\right| \leq C_{1}$ for any $n \geq 0$ and $0 \leq n_{0} \leq n$.

Proof: Let $D(z)$ be the Z-transform of $D(j)$. We have,

$$
D(z)=H_{1}(1) V(z)-\hat{V}_{1}(z)=\left(H_{1}(1)-z^{-n_{1}} H_{1}(z)\right) V(z)
$$

Because $z=1$ is a zero point of $H_{1}(1)-z^{-n_{1}} H_{1}(z)$, we can write the above equation as:

$$
D(z)=\left(1-z^{-1}\right) H(z) V(z),
$$

where $H(z)$ is still causal and stable. Now, define $Y(n)=$ $\sum_{i=0}^{n} D(i)$. We have,

$$
Y(z)=\frac{1}{1-z^{-1}} D(z)=H(z) V(z)
$$

Because $V(n)$ is bounded and $H(z)$ is a stable linear system, we know that $Y(n)$ will also be bounded. So, $\sum_{j=n_{0}+1}^{n} D(j)=$ $Y(n)-Y\left(n_{0}\right)$ is also bounded, i.e, there exists a constant $C_{1}$ such that for any $n \geq 0$ and $0 \leq n_{0} \leq n$,

$$
\left|\sum_{j=n_{0}+1}^{n} D(j)\right| \leq C_{1}
$$

Now, we can prove Theorem 1. First, we define $\Delta(j)=$ $V(j)-\hat{V}_{1}(j)$

$$
\begin{gathered}
\Delta(j)=V(j)-\hat{V}_{1}(j)=(V(j)-\bar{V})-\left(\hat{V}_{1}(j)-\bar{V}\right) \\
=D(j)+\left(1-H_{1}(1)\right)(V(j)-\bar{V}) . \\
a(j)+V(j)-\mu=a(j)+\hat{V}_{1}(j)+\Delta(j)-\mu \\
=a(j)+\hat{V}_{1}(j)+D(j)+\left(1-H_{1}(1)\right)(V(j)-\bar{V})-\mu \\
=a(j)+\hat{V}_{1}(j)-\mu^{\prime}+D(j)+\left(1-H_{1}(1)\right)(V(j)-\mu),
\end{gathered}
$$

where $\mu^{\prime}=H_{1}(1) \mu+\left(1-H_{1}(1)\right) \bar{V}$.

$$
\begin{aligned}
q(n)= & \sup _{0 \leq n_{0} \leq n}\left\{\sum_{j=n_{0}+1}^{n}(a(j)+V(j)-\mu)\right\} \\
\leq & \sup _{0 \leq n_{0} \leq n}\left\{\sum_{j=n_{0}+1}^{n}\left(a(j)+\hat{V}_{1}(j)-\mu^{\prime}\right)\right\} \\
& +\sup _{0 \leq n_{0} \leq n}\left\{\sum_{j=n_{0}+1}^{n} D(j)\right\} \\
& +\left(1-H_{1}(1)\right) \sup _{0 \leq n_{0} \leq n}\left\{\sum_{j=n_{0}+1}^{n}(V(j)-\mu)\right\} \\
\leq & q_{2}^{\prime}(n)+\sup _{0 \leq n_{0} \leq n}\left\{\sum_{j=n_{0}+1}^{n} D(j)\right\}+\left(1-H_{1}(1)\right) q_{0}(n) .
\end{aligned}
$$

If $H_{1}(1) \geq \frac{p \mu-\bar{V}}{\mu-V}$, we will have $\mu^{\prime} \geq p \mu$. From Lemma 2 we know,

$$
q_{2}^{\prime}(n)=q_{1}^{\prime}(n)=\sup _{0 \leq n_{0} \leq n}\left\{\sum_{j=n_{0}+1}^{n}\left(\hat{V}_{1}(j)-\mu^{\prime}\right)\right\} .
$$

$$
\begin{aligned}
\hat{V}_{1}(j)-\mu^{\prime}= & \left(\hat{V}_{1}(j)-\bar{V}\right)-H_{1}(1)(V(j)-\bar{V}) \\
& +H_{1}(1)(V(j)-\bar{V})+\bar{V}-\mu^{\prime} \\
= & -D(j)+H_{1}(1)(V(j)-\mu)
\end{aligned}
$$

So,

$$
\begin{aligned}
q_{2}^{\prime}(n)= & q_{1}^{\prime}(n)=\sup _{0 \leq n_{0} \leq n}\left\{\sum_{j=n_{0}+1}^{n}\left(\hat{V}_{1}(j)-\mu^{\prime}\right)\right\} \\
\leq & \sup _{0 \leq n_{0} \leq n}\left\{\sum_{j=n_{0}+1}^{n}-D(j)\right\} \\
& +H_{1}(1) \sup _{0 \leq n_{0} \leq n}\left\{\sum_{j=n_{0}+1}^{n}(V(j)-\mu)\right\} \\
\leq & -\inf _{0 \leq n_{0} \leq n}\left\{\sum_{j=n_{0}+1}^{n} D(j)\right\}+H_{1}(1) q_{0}(n) .
\end{aligned}
$$$$
q(n) \leq q_{2}^{\prime}(n)+\left(1-H_{1}(1)\right) q_{0}(n)
$$$$
+\sup _{0 \leq n_{0} \leq n}\left\{\sum_{j=n_{0}+1}^{n} D(j)\right\}
$$$$
\leq q_{0}(n)+\sup _{0 \leq n_{0} \leq n}\left\{\sum_{j=n_{0}+1}^{n} D(j)\right\}
$$$$
-\inf _{0 \leq n_{0} \leq n}\left\{\sum_{j=n_{0}+1}^{n} D(j)\right\}
$$

$$
\leq q_{0}(n)+2 C_{1}
$$

\section{PROOF OF THEOREM 2}

First, we let $Q_{i}(n)$ denote the queue length of a queue with $N a_{i}\left(n-n_{i}\right)+V(n)$ as the input traffic rate and $\mu$ as output link rate. From the definition of $a_{i}(n)$ and Theorem 1, we know, there exists a constant $C_{i}$ such that

$$
Q_{i}(n) \leq q_{0}(n)+2 C_{i},
$$

where $q_{0}(n)$ is the queue length of a queue without NRT traffic.

Because the input rate to $q$ is $a(n)+V(n)$, we have,

$$
\begin{aligned}
q(n) & =\sup _{0 \leq n_{0} \leq n}\left\{\sum_{j=n_{0}+1}^{n}(a(j)+V(j)-\mu)\right\} \\
& =\sup _{0 \leq n_{0} \leq n}\left\{\sum_{j=n_{0}+1}^{n}\left(\sum_{i=1}^{N} a_{i}\left(j-n_{i}\right)+V(j)-\mu\right)\right\} \\
& =\sup _{0 \leq n_{0} \leq n}\left\{\frac{1}{N} \sum_{i=1}^{N} \sum_{j=n_{0}+1}^{n}\left(N a_{i}\left(j-n_{i}\right)+V(j)-\mu\right)\right\} \\
& \leq \frac{1}{N} \sum_{i=1}^{N}\left(\sup _{0 \leq n_{0} \leq n}\left\{\sum_{j=n_{0}+1}^{n}\left(N a_{i}\left(j-n_{i}\right)+V(j)-\mu\right)\right\}\right) \\
& =\frac{1}{N} \sum_{i=1}^{N} Q_{i}(n) \leq \frac{1}{N} \sum_{i=1}^{N}\left(q_{0}(n)+2 C_{i}\right) \leq q_{0}(n)+2 C,
\end{aligned}
$$

where $C=\max _{1 \leq i \leq N} C_{i}$. 